\title{
Prevención del abandono escolar temprano. Aportaciones desde el Trabajo Social ${ }^{1}$ \\ Prevention of Early School Leaving. Contributions from Social Work
}

\author{
Ana Cristina Ruiz-Mosquera* \\ María de las Olas Palma-García**
}

\section{Resumen}

El Abandono Escolar Temprano constituye un problema social y, a la vez, individual que impide el desarrollo personal, social y académico de cada uno de los estudiantes implicados y deriva en la pérdida de capital humano necesario para el conjunto de la sociedad. Ante esta realidad los centros escolares y, más concretamente, sus profesionales han de saber anticiparse a los efectos que este fenómeno conlleva, incorporando su prevención como prioridad estratégica. Con el objetivo de conocer la influencia de la intervención desde el Trabajo Social sobre la prevención del abandono escolar temprano, se lleva a cabo el presente estudio en el que se analizan seis casos de jóvenes detectados en riesgo de desvinculación del sistema educativo. Tras realizar la descripción de cada caso, se analiza la intervención desarrollada por el Equipo de Intervención Psicosocial de sus centros escolares, atendiendo a los diferentes factores individuales, familiares y sociales que influyen en esta situación. Los resultados muestran la efectividad de las intervenciones preventivas realizadas por

${ }^{1}$ El presente artículo forma parte del proyecto de investigación en curso "Fenómeno NEET: jóvenes NiNi en vía de exclusión social" del programa de doctorado de la Universidad de Málaga.

* Máster en Investigación e Intervención Social y Comunitaria, y Doctoranda del programa de ciencias jurídicas y sociales de la Universidad de Málaga. Málaga, España. Correo electrónico: acristinaruizmosquera@gmail.com.

${ }^{* *}$ Licenciada en Ciencias Políticas y Sociología de la Universidad Pontificia de Salamanca, Diplomada en Trabajo Social y Doctora por la Universidad de Málaga. Profesora de Trabajo Social y Servicios Sociales de la Universidad de Málaga. Málaga, España. Correo electrónico:mpalma@uma.es.

Universidad del Valle. Cali, Colombia. Recibido: 08/06/2018 Aprobado: 08/11/2018

ISSN: 0122-1213 ISSN-e: 2389-993X Doi: 10.25100/prts.v0i27.6569] 
los profesionales del Trabajo Social a través de la atención individualizada, familiar y grupal, así como de la coordinación y del trabajo en equipo.

Palabras clave: Abandono escolar; Jóvenes en riesgo; Exclusión social; Intervención psicoeducativa.

\section{Abstract}

Early School Leaving is a social and individual problem which block up the personal, community and academic development of each students involved and the results for society is the lost of human capital. Focused in this reality, schools and, specifically, their professionals must anticipate the effects that this phenomenon entails, incorporating prevention as a strategic priority. With the aim of knowing the influence of the Social Work intervention in order to prevent early school leaving, the present study analyzes six cases of young people who were at risk of drop out from the educational system. After describing each case, the intervention developed by the Psychosocial Intervention Team of their schools is analyzed, taking into account the individual, family and social factors that influence this situation. The results show the effectiveness of preventive interventions carried out by Social Work professionals through individual, family and group care, as well as the coordination and teamwork

Keywords: School dropout; Social problem; Social exclusion; Psychoeducational intervention.

Sumario: 1. Introducción; 2. Metodología; 3. Resultados; 4. Discusión; 5. Conclusiones; 6. Referencias bibliográficas.

\section{Introducción}

El abandono escolar temprano (EAT) está protagonizado por la población de 18 a 24 años que, pudiendo haber completado la educación obligatoria, no sigue ninguna formación específica posterior y se desvincula del sistema educativo, lo que repercute de forma directa en su desarrollo personal y social. Ante dicha realidad, con este artículo queremos revalorizar la figura del trabajador social en el ámbito educativo, haciendo visible su función 
dentro de los equipos interdisciplinares que actúan en los centros escolares y sus actuaciones específicas para la prevención del AET.

Una de las principales preocupaciones de las políticas educativas a escala mundial es el AET (Przybylski, 2014). En su proyección europea, se presenta como prioridad central de la Estrategia de Educación y Formación 2020 (Consejo de la Unión Europea, 2009) con la que se espera reducir a un máximo del 10\% el porcentaje de jóvenes que abandonan prematuramente los sistemas educativos. En el caso español (contexto en el que se realiza este estudio) el porcentaje de AET ha ido disminuyendo en los últimos años pero se hace patente que el sistema educativo no es capaz de absorber y modificar las distintas problemáticas que dificultan la consecución de los objetivos que se plantean desde la institución escolar (Roselló, 1998). Prueba de ello es que a finales del año 2016 el porcentaje de AET se mantenía en un 19,4\%, cifra superior al objetivo marcado por la Estrategia Europea para el 2020 que se traduce en una importante cantidad de jóvenes desvinculados del sistema educativo.

Previo al abandono, los jóvenes escolares suelen presentar indicadores asociados a problemáticas de disciplina en los centros, al absentismo o al fracaso escolar (Sánchez, 2016). De hecho, el abandono escolar precoz es un paso más en una trayectoria de "desenganche" progresivo de la institución escolar (González-González, 2006). De esta forma, ante el AET es posible identificar determinados factores que lo favorecen, tanto relacionados con las características personales de los estudiantes, como con las circunstancias sociales que lo rodean. En general, los jóvenes con AET presentan falta de motivación por su desarrollo personal y social, desconocimiento sobre el futuro y desinterés por continuar formándose de una forma más específica (Sánchez, 2016). Respecto al género, existe el doble de probabilidad de que los niños abandonen la educación con muy baja o con ninguna titulación si lo comparamos con las niñas, confirmando que el abandono prematuro tiene más incidencia en los alumnos que entre las alumnas (Traag y Van der Velden, 2011). También inciden otras causas de tipo sociocultural, como la pérdida de valor de los estudios ante la no garantía de un puesto de trabajo en el futuro ni una adecuada posición en la sociedad (Comisión Europea/EACEA/Eurydice/Cedefop, 2014). Las 
condiciones del mercado laboral pueden afectar claramente a la decisión de los jóvenes de abandonar sus estudios, como factor "incentivador" o "disuasorio" del proceso. En los años previos a la crisis económica iniciada en el 2008, pudimos observar cómo grandes oportunidades de empleo de baja cualificación (como el turismo o la construcción) motivaba a los jóvenes a abandonar la educación de forma prematura para mejorar la situación económica de su familia o para poder ser más independientes.

La relación entre inestabilidad y/o precariedad laboral y el grado de implicación del entorno con el centro escolar es evidente (Pelegrí-Viaña, Mata-Romeu y Juliá-Tarveira, 2016), mostrándose como otro factor a tener en cuenta en el AET, la convivencia con desigualdades, es decir, los jóvenes que se desarrollan en entornos socioeconómicos y culturales desfavorecidos o que parten de otra cultura, idioma y características. Estos jóvenes encuentran dificultades añadidas para obtener éxito dentro del sistema educativo. Según indica el Parlamento Europeo (2011) en su informe Reducing Early School Leaving in Europe las mayores tasas de abandono escolar temprano las presenta la juventud inmigrante y el riesgo de protagonizar este proceso es especialmente alto en algunas minorías étnicas.

De forma central, la familia reúne gran parte de factores relacionados con el abandono escolar temprano (Comisión Europea/EACEA/Eurydice/ Cedefop, 2014). Es el primer agente de socialización de las personas, por lo que su influencia directa en la transformación educativa del individuo es fundamental. En el ámbito familiar se generan pautas culturales, se forjan actitudes y se fomenta la adquisición de hábitos fundamentales. Numerosos estudios han puesto de manifiesto que en la medida en la que los padres y las madres tienen menos nivel de estudios y profesiones menos cualificadas, el rendimiento de sus hijos es inferior (Martínez-Gutiérrez y Niemela, 2010). A su vez, factores como la inestabilidad o el modo de vida familiar, la monoparentalidad, las malas condiciones de vida, la salud física y mental y la violencia doméstica pueden aumentar las probabilidades de abandono prematuro de los estudios (Parlamento Europeo, 2011). Otros factores relacionados con la familia, como las relaciones entre los padres y el hijo o hija también pueden desempeñar un papel importante. Más grave 
aún resulta la falta de interés por los estudios de sus hijos, de implicación en el desarrollo de la vida escolar, de apoyo durante el proceso y de compromiso familiar (Comisión Europea, 2013). Todas estas situaciones influyen de forma directa en la motivación e interés que los individuos puedan desarrollar para continuar su formación específica una vez que ésta deja de ser obligatoria.

Por último, es necesario destacar otras causas que se generan y se relacionan con el propio sistema educativo. Son factores derivados fundamentalmente de la falta de coordinación docente y de trabajo en equipo entre quienes tienen relación directa con los estudiantes o de la ausencia de tutorización efectiva, personalizada e individualizada atendiendo a las características de cada estudiante. El currículum y las metodologías empleadas también juegan un papel crucial, al ser en ocasiones alejadas de los intereses y necesidades de los alumnos y obsoletas en su implementación. Por último, acciones como la repetición de curso, la segregación socioeconómica de los centros educativos y la diferenciación precoz de itinerarios, son a su vez factores causales en el incremento de los porcentajes de abandono escolar temprano (Comisión Europea/EACEA/ Eurydice/Cedefop, 2014). Junto a estos factores, van a ser de igual forma determinantes en el desarrollo del AET las percepciones y prácticas del profesorado y de otros profesionales implicados y el tipo de orientación ofrecida a los estudiantes (García-Gracia, 2005).

Ante esta realidad, es fundamental el desarrollo de estrategias de intervención socioeducativas en los centros escolares que sean capaces de detectar y abordar las causas previas al abandono escolar temprano, elevando y cualificando el nivel de formación de los jóvenes más allá de la escolarización obligatoria. La función de los centros educativos pasa por acompañar al menor y a su familia en muchos de los avatares que suceden en torno a la educación y socialización (Juliá-Tarveira, Mata-Romeu y Pelegrí-Viaña, 2016) siendo los profesionales de lo social los encargados de diseñar estrategias durante el proceso de acompañamiento.

En estas estrategias, el Trabajo Social encuentra espacios de actuación propios, al poder intervenir sobre los factores que promueven el AET, identificando circunstancias personales, familiares o comunitarias 
que inciden en ello y desarrollando mecanismos para su evaluación y prevención. Se trata de profesionales que vienen a cubrir lagunas existentes en el propio sistema educativo tanto en su desarrollo como en su acción preventiva (Roselló, 1998).

Sin embargo, lo cierto es que en la actualidad en el contexto educativo, la figura de los trabajadores sociales se encuentra en un proceso de maduración escasamente reconocido, principalmente en cuanto a sus funciones y quehacer profesional (Quiroz y Sepúlveda, 2016) que podríamos sintetizarlas de acuerdo con Roselló (1998) en el establecimiento y fortalecimiento de las relaciones entre el medio escolar, el familiar y el comunitario, la integración escolar y social de los menores, y la intervención sobre aquellos obstáculos que puedan encontrar durante su desarrollo integral.

De acuerdo con Bravo (2002) para que la prevención del abandono escolar temprano sea eficaz y eficiente, las problemáticas de absentismo y fracaso escolar deben ser intervenidas desde la óptica de los profesionales del trabajo social pero ¿existen ejemplos de buenas prácticas de prevención del abandono escolar temprano desde la intervención social?, ¿cómo intervienen los profesionales del trabajo social en estos casos en la actualidad?

Esta investigación nace con el objetivo de avanzar en el conocimiento y explicación del abandono escolar temprano, a la vez de explorar las aportaciones del Trabajo Social en su prevención. El presente estudio se dirige a analizar las intervenciones psicosociales de carácter preventivo desarrolladas en centros escolares que cuentan con la figura de trabajadores sociales.

\section{Metodología}

\section{Enfoque metodológico}

Este estudio se ha llevado a cabo utilizando la metodología cualitativa, concretamente desde el método de estudio de casos múltiples (Yin, 2013) con el que se han descrito los distintos contextos sociales y educativos, las situaciones de los estudiantes, los factores que los situaban en riesgo 
de abandono escolar temprano y la intervención de los profesionales del trabajo social para su prevención.

\section{Diseño del estudio}

Esta aproximación cualitativa nos ha permitido conocer de cerca los procesos educativos que se experimentan en el día a día en los centros escolares durante el tercer trimestre del curso 2016/17, pudiendo así explorar la intervención realizada por el equipo de intervención psicosocial en relación a la gestión y prevención de los casos de riesgo de abandono escolar. Para ello se seleccionaron dos centros escolares concertados con Educación Secundaria Obligatoria en la ciudad de Málaga que cumplían con el criterio de contar con la figura del profesional del trabajo social en su plantilla como parte del Equipo de Intervención Psicosocial (EIPS) y que aceptaron formar parte de nuestro estudio.

\section{Participantes}

Los centros escolares participantes son centros con alumnado preferente de clase media, cuyas familias presentan un capital cultural medio y óptimas expectativas educativas sobre sus hijos/as. Ambos centros tienen además una buena valoración en sus zonas de referencia, recibiendo cada curso una elevada demanda educativa. El Equipo de Intervención Social en estos centros escolares está compuesto por la trabajadora social (compartida para ambos centros) y las psicólogas con funciones de orientación (una en cada centro).

La selección de casos de estudiantes como unidad de análisis en ambos centros se ha basado en los siguientes criterios (ver tabla 1): que los jóvenes perteneciesen al segundo ciclo de educación secundaria obligatoria y que se encontraran en riesgo de abandono escolar debido a la concurrencia de factores personales, escolares y sociales. Los estudiantes participantes han sido: 
Tabla 1. Datos sobre participantes

\begin{tabular}{cccc}
\hline $\mathbf{N}^{\mathbf{0}}$ de caso & Sexo & Curso & Edad \\
\hline 1 & Mujer & $4^{\circ} \mathrm{ESO}$ & 17 \\
\hline 2 & Hombre & $4^{\circ} \mathrm{ESO}$ & 16 \\
\hline 3 & Mujer & $3^{\circ} \mathrm{ESO}$ & 15 \\
\hline 4 & Hombre & $3^{\circ} \mathrm{ESO}$ & 15 \\
\hline 5 & Hombre & $3^{\circ} \mathrm{ESO}$ & 17 \\
\hline 6 & Hombre & $3^{\circ} \mathrm{ESO}$ & 15 \\
\hline
\end{tabular}

Fuente: elaboración propia

\section{Procedimiento}

El seguimiento y análisis de casos se realizó desde el mes de abril hasta julio de 2017. En primer lugar se realizó una solicitud a la dirección de ambos centros escolares en la que se indicaban los objetivos de la investigación. También se solicitó permiso por escrito a las familias de los menores sujetos de estudio. La selección de casos $(\mathrm{N}=6)$ se llevó a cabo tras la finalización de la reunión de evaluación del segundo trimestre escolar (abril 2017), contando con la colaboración de tutores y tutoras, orientadoras de cada centro y de la trabajadora social. Con los jóvenes seleccionados se mantiene seguimiento tres días por semana, a través de entrevistas, reuniones, talleres y acciones de derivación. La recogida de datos fue realizada mediante el instrumento del diario de campo, herramienta que ha ayudado a sistematizar los resultados. Posteriormente se procedió al análisis inductivo de estos datos cualitativos.

\section{Resultados}

En el siguiente apartado se describen los casos estudiados explorando las diferentes intervenciones realizadas para gestionar los riesgos de abandono escolar temprano. La heterogeneidad de los estudiantes y de los factores de riesgo que presentan ha permitido el desarrollo de intervenciones diferentes, diseñadas de acuerdo a las características individuales, situación familiar y desarrollo escolar y social de cada sujeto. 


\section{Caso 1}

La estudiante tiene 17 años y en la actualidad cursa $4^{\circ}$ curso de Educación Secundaria Obligatoria (ESO). En su trayectoria escolar los suspensos en Lengua e Inglés han sido continuos, repitiendo segundo de educación primaria por dificultades en el proceso lector, aun cuando no presentaba otros problemas a la hora de adquirir conocimientos. La situación de riesgo de abandono escolar temprano se hace patente cuando comienza a suspender todas las materias, manifestando que tiene dificultades de concentración y que "no puede parar de llorar", no quiere acudir a clase. Tras mantener entrevistas con ella y con sus padres es derivada a salud mental, donde se detecta un estado emocional muy bajo y sentimientos de depresión e inutilidad que comienzan a ser abordados de forma especializada.

Intervención: Desde el Equipo de Intervención Psicosocial del centro escolar se diseña un proyecto de orientación educativa individualizada con esta alumna. En las entrevistas realizadas por la trabajadora social se exploran sus motivaciones y competencias, identificándose su potencial y trabajando para que ella misma lo reconozca. Se constata que se trata de una niña muy luchadora, sociable y con buenas relaciones familiares (la separación de sus padres no le ha supuesto un inconveniente en su desarrollo personal ni académico). Aun así, necesita ayuda, seguimiento, motivación, apoyo y control en la etapa no voluntaria de escolarización que comienza el próximo curso. En una de las entrevistas finales manifiesta querer continuar estudiando para conseguir un título que le permita trabajar en el mundo de la cocina. Se le orienta sobre la posibilidad de la Formación Profesional Básica de Cocina y Restauración.

\section{Caso 2}

Se trata de un estudiante de 16 años que cursa $4^{\circ}$ de ESO. A mediados de curso, concretamente a la vuelta de navidades comienza a faltar a clase, teniendo todas las asignaturas suspensas. Es un niño frío, con dificultades para expresar sus sentimientos. En cuanto a su situación familiar, convive con su madre que está separada y 
casada de nuevo. El menor manifiesta no tener buena relación con la nueva pareja de su madre y pasar por ello mucho tiempo en casa de su abuela. Durante el curso continúa con todas las asignaturas suspensas y muestra dificultades en las relaciones sociales.

Intervención: Debido al bajo rendimiento académico y a las continuas faltas de asistencia, el tutor se pone en contacto con la madre quien inicialmente no se muestra colaboradora. Ante ello, la trabajadora social diseña y pone en marcha un proyecto de intervención individualizada. Mantiene reuniones periódicas con la madre, en las que se aborda la situación familiar, situando en el centro de la intervención la mejora de la relación y comunicación con su hijo. A lo largo de la intervención se detectan los intereses formativos del menor, relacionados con la mecánica, y se aborda con la madre las opciones más adecuadas para la garantía de la continuidad de sus estudios. Tras orientarla e informarla sobre los intereses y potencialidades de su hijo, decide realizar matrícula para el próximo curso en Formación Profesional Básica de Mantenimiento de Vehículos.

\section{Caso 3}

Se trata de una alumna de 15 años matriculada en $3^{\circ}$ de ESO que llega al centro escolar por primera vez. En la actualidad convive con su madre y abuelos. Carece de habilidades sociales, presenta dificultad para la comunicación e insuficiente red de amigos. Se conoce que en el centro escolar anterior presentaba absentismo escolar no habiendo sido hasta el momento derivada al sistema de Servicios Sociales. En esta ocasión, a partir de las numerosas faltas de asistencia presentadas al comienzo del curso y de las dificultades en la colaboración familiar, se pone en conocimiento de los Servicios Sociales. Los días que asiste a clase se observa en la alumna un aspecto de abandono y descuido, lo que provoca el rechazo entre sus compañeros y compañeras.

Intervención: En paralelo a la derivación a Servicios Sociales, el Equipo de Intervención Psicosocial del centro diseña y comienza a desarrollar actuaciones de acompañamiento familiar. La trabajadora

Prospectiva. Revista de Trabajo Social e intervención social No. 27, enero-junio 2019: pp. 139-158 (c) (1) () () 
social contacta con la familia y mantiene reuniones con la madre y la abuela, en las que se aborda la situación familiar y personal de la alumna. A través de ello se conoce que la joven no acude a clases por voluntad propia, la cual es aceptada por sus mayores, cuando han intentado convencerla se enfada y se encierra en su habitación. Se realizan tareas de coordinación con la trabajadora social de servicios sociales proponiendo a la familia un plan de trabajo para la reducción del absentismo, con asistencia a un taller de habilidades sociales y asertividad impartido en el centro escolar, con acciones de tutorización periódica y orientación a la alumna. Se consigue la firma de un compromiso de asistencia por el que la joven acepta asistir a diario al centro. A lo largo de la intervención, la alumna manifiesta encontrarse más a gusto en clase y con sus compañeros. Mejora su respuesta ante situaciones sociales y asiste diariamente a clase.

\section{Caso 4}

Alumno de $3^{\circ}$ de ESO, con 15 años de edad. Desde principio de curso tiene muchas faltas de asistencia y en el primer trimestre suspende 6 asignaturas. En el segundo trimestre continúa suspendiendo, a pesar de que no presenta dificultades en el aprendizaje. Llegó al centro este curso, no teniéndose hasta ese momento ninguna información previa relevante sobre su trayectoria escolar.

Intervención: Se inicia intervención desde el Equipo del centro abordando en una primera entrevista individualizada con el alumno las causas de sus faltas de asistencia a clase. En la entrevista, la trabajadora social detecta que el menor se siente incómodo en clase, con miedo a que lo juzguen por su orientación sexual como hacían en el centro escolar del que vino a terminar la ESO. Además, él considera que habla muy rápido y cree que por ello nadie lo entiende, lo que le lleva a no intervenir en clase ni formar parte de las dinámicas. Se diseña un programa de comunicación para el alumno, llevado a cabo en el centro por el aula de Audición y Lenguaje. En paralelo se diseña una estrategia grupal en el aula, en la que el tutor tratará de hacerlo partícipe en los temas que se tratan en clase, dándole la 
palabra para que intervenga en público y trabajando la diversidad. Comienza a establecer vínculos con sus compañeros a partir de actividades que le ayudan a definirse, a conocerse un poco más, estos talleres son diseñados por el Equipo de Intervención Psicosocial. A lo largo de la intervención comienza a mejorar en sus calificaciones y reconoce sentirse más a gusto. La trabajadora social del centro lo incluye en uno de sus talleres en los que se trabaja la diversidad y la inclusión. En estos talleres también pone en práctica las habilidades de comunicación aprendidas en el aula de Audición y Lenguaje.

\section{Caso 5}

Alumno de 17 años matriculado por segunda vez en $3^{\circ}$ de la ESO. Vive con sus padres y dos hermanas. Inicia el curso con normalidad, pero a los cuatro días comienza a faltar a clase argumentando motivos de salud. El contacto inicial con la familia pone de manifiesto que el menor pasa los días completos jugando a videojuegos sin control familiar.

Intervención: El Equipo de Intervención Psicosocial propone a la familia la participación de su hijo en un taller de inteligencia emocional a desarrollar en el centro escolar. Sin embargo el menor sigue sin acudir a clase y la familia deja de colaborar con las actuaciones planteadas desde los profesionales. Por la edad del menor, su escolarización ya no es obligatoria, poniéndose aun así en conocimiento de los Servicios Sociales las circunstancias familiares y de no control sobre el uso de videojuegos que rodean al alumno. En coordinación con Servicios sociales se plantea su incorporación en un programa especializado hacia jóvenes sobre adicciones a videojuegos. A finales del tercer trimestre el menor continúa sin asistir al centro escolar, por lo que no supera el curso.

\section{Caso 6}

Alumno de 15 años matriculado en $3^{\circ}$ de ESO. Es alumno del centro desde que inicia su escolarización. Los padres están separados desde hace 8 años y él convive con la madre y una hermana. Pasa semanas sin asistir a clase y cuando acude presenta un comportamiento 
disruptivo y carencia de habilidades sociales. Manifiesta que se encuentra cansado y que no entiende lo que se explica en clase. $\mathrm{Su}$ asistencia es discontinua y no sigue el ritmo de las clases. En coordinaciones previas mantenidas con Servicios Sociales se conoce que el padre ha iniciado tratamiento de deshabituación al consumo de alcohol. La fiscalía de menores ha pedido información al centro en relación a sus hábitos de estudio, comportamiento y relación de los padres con el centro escolar.

Intervención: Se abre el protocolo de absentismo escolar pero no es posible establecer compromiso de asistencia ya que los padres no responden a las numerosas citas que se le proponen desde el EIPS. Los días que el menor acude a clase, la trabajadora social interviene con él a través de un taller de habilidades sociales en el que el menor va expresando su situación. Se realiza un seguimiento diario en el que va facilitándose la relación con el menor, quien comienza a reconocer que en ocasiones quiere estudiar para conseguir un buen futuro pero que su situación en casa no se lo permite. Se planifican contactos con la familia que no llegan a producirse, la madre nunca se encuentra en disposición de hablar y el padre está bajo tratamiento por lo que tampoco puede prestar atención a la situación que atraviesa su hijo. El caso es derivado a Servicios Sociales.

En la tabla 2 se muestran los resultados finales de las intervenciones llevadas a cabo con cada alumno/a tras ser evaluadas por el equipo. Se utilizan como indicadores de evaluación: la continuidad en el sistema educativo, el abandono escolar temprano o la derivación del caso a otros servicios. Los resultados obtenidos son:

Tabla 2. Resumen de resultados

\begin{tabular}{cl}
\hline $\mathbf{N}^{\mathbf{0}}$ de caso & \multicolumn{1}{c}{ Resultado de la Intervención } \\
1 & Continúa Sistema Educativo: FP Básica \\
2 & Continúa Sistema Educativo: FP Básica \\
3 & Continúa Sistema Educativo: $4^{\circ}$ ESO \\
4 & Continúa Sistema Educativo: $4^{\circ}$ ESO \\
5 & Abandono Escolar Temprano \\
6 & Derivado a Servicios Sociales \\
\hline
\end{tabular}

Fuente: elaboración propia 
Según se observa en la tabla anterior, la prevención del abandono escolar ha sido efectiva en cinco de los seis casos detectados y acompañados desde la intervención del Equipo de Intervención Psicosocial. Solo en un caso se ha producido el abandono escolar.

\section{Discusión}

En el seguimiento de casos analizados en el presente estudio se ha corroborado que el abandono escolar temprano (AET) se relaciona con diferentes factores, principalmente de tipo familiar, escolar y social (Comisión Europea/EACEA/Eurydice/Cedefop, 2014) y que como indicaba Roselló (1998) es decisiva la figura del trabajador social para "detectar aquellas condiciones, ya sean personales o sociales, que están dificultando el proceso de desarrollo escolar de los estudiantes y su adaptación al ámbito escolar" (p. 239). También se ha puesto de manifiesto, tal como señalan Traag y Van der Velden (2011), la mayor presencia de estos factores entre alumnos chicos, identificándose entre los sujetos participantes mayor riesgo de abandonar la escuela en ellos frente a las alumnas. En cualquier caso ante el AET existen motivos internos o externos al sujeto, y aun cuando la decisión última pueda corresponder a cada estudiante, la intervención del entorno (familia, profesionales del centro y de otros sistemas de protección) va a ser determinante sobre ella, tal como muestran los resultados.

En primer lugar, para prevenir el abandono escolar es fundamental que el Equipo de Intervención Psicosocial realice un seguimiento individualizado del alumnado desde sus inicios en la educación secundaria obligatoria, conociendo su trayectoria escolar previa. Solo de esta forma es posible anticiparse y detectar a tiempo indicadores relacionados con el proceso que lleva al AET (absentismo escolar, calificaciones, circunstancias psicosociales, etc.). De acuerdo con González-González (2006), el abandono total de la escuela se produce tras un proceso de "desenganche" progresivo en el que siempre aparecen oportunidades para la intervención.

En el presente estudio, las experiencias escolares previas y la tendencia al absentismo se han mostrado como indicadores frecuentes de riesgo de 
AET en los casos analizados. La mayoría de ellos presentaban faltas de asistencia, mantenidas en el tiempo o sobrevenidas, que siendo incluso justificadas por los padres, han resultado ser estrategias familiares para evitar enfrentarse al problema. De igual forma, el cambio negativo en los resultados académicos alcanzados por los jóvenes a lo largo del curso ha sido otro indicador clave para la prevención del AET. Ante estos indicadores, la rapidez en su detección ha permitido al equipo interventor desarrollar estrategias efectivas de abordaje y prevención con cada uno de los casos identificados. En todos los supuestos, la intervención se ha iniciado explorando los factores actitudinales que sitúan a los jóvenes en riesgo de abandono escolar y las verdaderas motivaciones individuales de cada estudiante. Ha sido crucial conocer sus características individuales, aquellos datos más personales que hacen a los estudiantes únicos, sus habilidades y su potencial, para desde ellas poder establecer una línea de trabajo que permita el máximo desarrollo de su vida personal y social. El instrumento protagonista en este tipo de intervención ha sido la entrevista, en la que se ha ido construyendo la relación de ayuda y orientación profesional que ha permitido al trabajador social desarrollar sus capacidades para valorar y comprender las posibilidades de cambio y oportunidades de las personas, familias y del propio centro escolar (Fombuena-Valero, 2012).

Junto al lugar central reconocido a cada alumno y alumna en las estrategias diseñadas, otro de los resultados a destacar de estas intervenciones ha sido la importancia de favorecer que también la familia entienda o recupere su espacio en este proceso. Como señalaba Fernández (2007) la escuela por sí sola no puede educar, necesita la colaboración del medio para poder llevar a cabo esta labor. En este sentido, las intervenciones se han dirigido a hacer partícipes a las familias del papel que estaban jugando como agentes imprescindibles en el desarrollo educativo de sus hijos, tratando de que formaran parte activa del itinerario que se estaba diseñando y reforzando al mismo tiempo la coordinación y comunicación con el centro escolar. De acuerdo con Bolívar (2006), los resultados han puesto de manifiesto que trabajar de modo conjunto, dentro de la escuela, con las familias y con otros actores de la comunidad facilita que los centros escolares puedan llevar a cabo su labor educativa. 
Por otro lado, junto a la intervención individualizada y diseñada como itinerario personalizado para cada estudiante, el desarrollo de estrategias grupales complementarias han sido claves para el logro de los objetivos de prevención del AET. En concreto, el desarrollo de actuaciones basadas en la intervención por programas (Martínez-Clares, 2002) tales como los talleres de habilidades sociales puestos en marcha por la trabajadora social han actuado como espacios de protección. Con las estrategias grupales se han trabajado aspectos relacionados con la diversidad y la inclusión del alumnado, ofreciendo espacios adaptados a cada alumno y por tanto, a la situación que atraviesa con las distintas problemáticas a las que se enfrenta.

Otro elemento fundamental en todas las intervenciones ha sido la coordinación entre los diferentes agentes que tienen papel activo en este proceso. De acuerdo con Roselló (1998) la intervención socioeducativa de los trabajadores sociales se debe realizar en y con la comunidad educativa, en constante contacto con el objetivo de garantizar el máximo aprovechamiento de los recursos sociales existentes. Esta estrategia ha permitido diseñar la intervención desde una mirada más amplia de la situación personal y familiar de los jóvenes que acuden a los centros.

Los resultados han evidenciado el efecto preventivo de la coordinación cuando ésta se establece de forma transversal entre todos los actores implicados: entre los profesionales del propio centro escolar, entre ellos con la familia de los estudiantes y entre otros agentes externos al ámbito educativo. El trabajo coordinado y la retroalimentación producida en el proceso, ha permitido que los estudiantes no se desvincularan del centro educativo, ofreciendo respuestas rápidas y eficaces para la prevención de situaciones de riesgo social (Rossell, 1999).

La intervención social en la escuela está protagonizada por una negociación constante, cuyo peso final recae sobre los estudiantes tras el proceso de orientación promovido por profesionales de lo social favoreciendo la integración escolar cuando esta se ve amenazada por distintos factores (Pelegrí-Viaña, Mata-Romeu y Juliá-Tarveira, 2016).

Como establecía Palma-García (2014) la educación hace referencia a una necesidad que acompaña a la persona a lo largo de su vida y que puede ser satisfecha desde distintos ámbitos en función de la dimensión 
que se le otorgue al concepto. Por este motivo ha resultado fundamental la orientación y educación (tanto psicológica como social) del entorno que acompaña en el desarrollo personal y social a estos niños/as. El ámbito familiar juega un papel crucial en la prevención del abandono escolar, si el entorno familiar valora la educación, transmitirá ese valor a sus hijos/as y trabajará para que continúe formando parte del sistema educativo. En el desarrollo de la educación confluyen todos los aspectos fundamentales de la persona que permitirá y motivará el formar parte activa de la sociedad en la que se están desarrollando, evitando la exclusión social de forma directa.

\section{Conclusiones}

Según los datos relativos al Abandono Escolar Temprano (AET) en nuestros contextos más próximos, nos encontramos ante un problema social y, a la vez individual, que impide el desarrollo personal, social y académico de cada uno de los estudiantes implicados y deriva en la pérdida de capital humano necesario para el conjunto de la sociedad. Ante esta realidad, los centros escolares y, más concretamente sus profesionales, han de saber anticiparse a los efectos que este fenómeno conlleva, situando la prevención del mismo, como prioridad estratégica entre sus objetivos.

El Abandono Escolar Temprano es el último paso de una trayectoria previa de dificultades, fracaso o bajo rendimiento escolar, bajas expectativas, factores actitudinales, insuficiente relación e implicación con el centro, absentismo y baja motivación. Todos ellos, indicadores que deben ser detectados y abordados desde la prevención. En este sentido, las respuestas más eficaces que han de llevarse a cabo desde los interventores educativos y sociales son aquellas que proporcionen a los estudiantes mayores oportunidades de aprendizaje y de experiencias positivas a su paso por los centros educativos, incorporando en dichas experiencias a todos los agentes implicados. Este compromiso global con todo el alumnado ha de ser a su vez particularizado de forma especial hacia aquellos estudiantes que se encuentren en situaciones de mayor riesgo de abandono escolar. La prevención del AET ha de responder, por tanto, a la heterogeneidad de todo el alumnado y a la individualidad de cada 
estudiante. Para ello, los trabajadores y trabajadoras sociales disponen de capacidades demostradas para impulsar la intervención preventiva a través de la atención individualizada, familiar, grupal, del trabajo en equipo, en red y con enfoque comunitario (Quiroga y Chagas-Lemos, 2018). Todas estas capacidades se muestran de gran aplicabilidad en el contexto del Trabajo Social escolar, al que el presente estudio quiere contribuir.

\section{Referencias bibliográficas}

Bolívar, A. (2006). Familia y escuela: dos mundos llamados a trabajar en común. Revista de Educación, 339, 119-146.

Bravo, M. (2002). El trabajo social en un equipo de orientación educativa y psicopedagógica. Intervención sociofamiliar: Especificidad en el ámbito educativo. Madrid: Centro de Apoyo al Profesorado de Vallecas.

Consejo de la Unión Europea. (2009). Marco Estratégico para la cooperación europea en el ámbito de la Educación y la Formación («ET 2020») Diario Oficial de la Unión Europea, 119/02. Recuperado de https://eur-lex.europa.eu/ legal-content/ES/TXT/PDF/?uri=CELEX:52009XG0528(01)\&from=ES.

Comisión Europea. (2013). Study on Educational Support to newly arrived migrant children. Final report. Luxembourg: Publications Office of the European Union.

Comisión Europea/EACEA/Eurydice/Cedefop. (2014). La lucha contra el abandono temprano de la educación y la formación en Europa: estrategias, políticas y medidas. Informe de Eurydice y Cedefop. Luxemburgo: Oficina de publicaciones de la Unión Europea.

Fernández, M. (2007). Educar es cosa de todos: escuela, familia y comunidad. En J. Garreta (ed.) La relación familia-escuela (pp. 13-32). Lleida: Universidad de Lleida.

Fombuena-Valero, J. (2012). El trabajo social y sus instrumentos. Elementos para una interpretación a piacere. Valencia, España: Nau Llibres.

García-Gracia, M. (2005). Culturas de enseñanza y absentismo escolar en la Enseñanza Secundaria Obligatoria: Estudio de casos en la ciudad de Barcelona. Revista de Educación, 338, 347-374.

González-González, M. (2006). Absentismo y abandono escolar: una situación singular de la exclusión educativa. REICE - Revista Electrónica Iberoamericana Sobre Calidad, Eficacia y Cambio En Educación, 4(1), 1-15.

Juliá-Tarveira, R, Mata-Romeu, A. y Pelegrí-Viaña, X. (2016). La participación familia-Escuela. Encuentros y desencuentros. Una visión desde los profesionales externos. Documentos de Trabajo Social, (Ed. Esp.), 135-143.

Prospectiva. Revista de Trabajo Social e intervención social No. 27, enero-junio 2019: pp. 139-158 (c) (i) () () 
Martínez-Clares, P. (2002). La orientación psicopedagógica: modelos y estrategias de intervención. Madrid: Ed. EOS. Instituto de Orientación Psicológica Asociados.

Martínez-Gutiérrez, B. y Niemela, R. (2010). Formas de participación de las familias y de la comunidad hacia el éxito educativo. Revista Educación y Pedagogía, 22(56), 69-78.

Palma-García, M. (2014). La atención social desde el sistema público de la educación en España. En Gómez, M.L. (dir). Estudio comparado de la provisión de los servicios sociales: España- Costa Rica. (Ed. Málaga), 173-193.

Parlamento Europeo. (2011). Reducing early school leaving in the EU. Study. Bruselas, Bélgica: Parlamento Europeo.

Pelegrí-Viaña, X., Mata-Romeu, A. y Juliá-Tarveira, R. (2016). Las profesiones sociales en los centros educativos. Funciones y expectativas. En D. Carbonero, E. Raya, N. Caparrós y C. Gimeno. (Coords.) Respuestas transdisciplinares en una sociedad global: aportaciones desde el Trabajo Social, (p. 74). Logroño: Universidad de La Rioja.

Przybylski, B.K. (2014). Unsuccessful in Education: Early school leaving. En D. Eibel, E. Rokicka \& J. Leaman (eds.) Welfare State at Risk. Rising inequality in Europe, (pp. 153-166). Alemania: Springer International Publishing.

Quiroga, V. y Chagas-Lemos, E. (2018). Atención a adolescentes y jóvenes de origen inmigrante en centros de Cáritas Diocesana de Barcelona: factores de riesgo y de protección. PROSPECTIVA. Revista de Trabajo Social e intervención social, (25), 67-90. doi:10.25100/prts.v0i25.4796.

Quiroz, S. y Sepúlveda, P. (2016). Importancia del trabajo social en la intervención socio-educativa de la educación sexual. Revista Ibero-Ameicana de Estudos em Educação, 11(esp.1), 456-466. Doi: 10.21723/RIAEE.v11.esp.1.p456.

Rossell, T. (1999). El equipo interdisciplinario. Revista de Servicios sociales y Política social, 46, 9-20.

Roselló, E. (1998). Reflexiones sobre la intervención del trabajador social en el contexto educativo. Alternativas. Cuadernos de Trabajo Social, 6, 233-258.

Sánchez, A. (2016). Aspectos académicos y personales que inciden en el abandono escolar temprano en educación secundaria obligatoria. (Tesis doctoral) Universidad Complutense de Madrid. Facultad de Educación. Departamento de Didáctica y Organización Escolar. Madrid, España. Recuperado de https:// eprints.ucm.es/42407/1/T38717.pdf.

Traag, T. \& Van der Velden, R.K.W. (2011). Early school-leaving in the Netherlands: the role of family resources, school composition and background characteristics in early school-leaving in lower secondary education. Irish Educational Studies, 30(1), 45-62. Doi: 10.1080/03323315.2011.535975. 
Yin, R.K. (2013). Case study research: Design and methods. London: SAGE publications.

Cómo citar:

Ruiz-Mosquera, A.C. y Palma-García, M. (2019). Prevención del Abandono Escolar Temprano. Aportaciones desde el Trabajo Social. Prospectiva. Revista de Trabajo Social e intervención social, (27), 139-158. Doi: 10.25100/prts. v0i27.6569.

Prospectiva. Revista de Trabajo Social e intervención social No. 27, enero-junio 2019: pp. 139-158 (c) (i) () () 